\title{
Comparación del crecimiento del camarón blanco en dos condiciones de estudio, salinidad óptima y salinidad cercana a cero
}

\author{
Comparison of white shrimp growth under two study conditions, \\ optimal salinity and salinity close to zero
}

\author{
Martha Lorena Roque Salinas ${ }^{1}$ \\ María Luisa Canales Machado ${ }^{2}$ \\ Octavio Josué Cáceres Quiroz ${ }^{3}$ \\ José Javier Flores Romero ${ }^{4}$ \\ Noelia Erlinda Cea Navas ${ }^{5}$ \\ Valeria Mercedes Hernández Dimas ${ }^{6}$
}

\section{Resumen}

En Nicaragua el cultivo del camarón blanco (Litopenaeus vannamei), es un producto hidrobiológico que aporta importantes divisas a la economía del país, el éxito obtenido en este rubro se debe a que empresarios y productores han incentivado, extendido y tecnificado las áreas productivas destinadas a la camaronicultura. Sin embargo, el crecimiento de esta industria se ha visto afectada por las incidencias de enfermedades virales y bacterianas, ocasionando problemática a nivel social y económica en el rubro, razón por la cual a lo largo de la evolución de la camaronicultura y de las enfermedades que la afectan, paralelamente se han realizado estudios con el fin de encontrar alternativas que minimicen el impacto de los patógenos en el cultivo, encontrándose cultivo del camarón en tierra adentro; práctica que se lleva a cabo en muchos países desarrollados y en los cuales se presentan resultados similares a la producción tradicional. La finalidad del estudio fue comparar el crecimiento del camarón blanco desde sistemas de cultivo tradicional y el de tierra adentro, a través de la implementación de dos tratamientos que consisten en someter a los organismos a salinidades óptimas $\left(\mathrm{T}_{1}\right)$ y salinidades cercanas a o $\left(\mathrm{T}_{2}\right)$, siendo esta la única variante en el manejo del cultivo durante un período de 49 días y con una densidad de 27org/ $\mathrm{m}^{2}$, obteniendo resultados de pesos finales de 17.75 gramos para el $\mathrm{T} 1 \mathrm{y}$ de 12 gramos para el $\mathrm{T}_{2}$, revelando que los factores físicos y químicos no afectaron el crecimiento de los camarones durante el estudio.

Palabras clave: Cultivo; camarón; crecimiento; salinidad.

\footnotetext{
Docente del Departamento de Acuícola de la UNAN-León. Email: martha.roque@ev.unanleon.edu.ni ORCID oooo-0002-9054-9640

Ingeniera Acuícola. Email: mcanalesmachado17@gmail.com ORCID oooo-ooo2-2963-4387

Ingeniero Acuícola. Email. octavio93@gmail.com ORCID oooo-ooo1-6762-7359

Ingeniero Acuícola. Email: josejavierfloresromero@hotmail.com ORCID oooo-0001-7133-7354

Docente del Departamento de Acuícola de la UNAN-León. Email: noelia.cea@ev.unanleon.edu.ni ORCID oooo-ooo1-7913-9459

Docente del Departamento de Acuícola de la UNAN-León. Email: valeria.hernandez@ev.unanleon.edu.ni ORCID oooo-0003-1864-0068
}

Recibido: 14/02/2020 Aprobado: 29/05/2020

Roque Salinas, M., Canales Machado, M., Cáceres Quiroz, 0., Flores Romero, J., Cea Navas, N., y Hernández Dimas, V. (2020). Comparación 


\section{Summary}

In Nicaragua, the cultivation of white shrimp (Litopenaeus vannamei) is a hydrobiological product that contributes significant foreign exchange to the country's economy. The success obtained in this area is due to the fact that businessmen and producers have encouraged, expanded and technified the productive areas meant to shrimp farming. However, the growth of this industry has been affected by the incidences of viral and bacterial diseases, causing problems at a social and economic level in the field, which is why throughout the evolution of shrimp farming and the diseases that they affect it, in parallel studies it have been carried out in order to find alternatives to minimize the impact of pathogens on the farming, finding shrimp growing inland; practice that it is carried out in many developed countries and in which similar results to traditional production are presented. The purpose of the study was to compare the growth of white shrimp from traditional farming systems and inland farming, through the implementation of two treatments that consist of subjecting organisms to optimal salinities ( $\left.\mathrm{T}_{1}\right)$ and salinities close to $\mathrm{o}\left(\mathrm{T}_{2}\right)$, being this the only variant in the management of the crop during a period of 49 days and with a density of 27 org / $\mathrm{m}^{2}$, obtaining results of final weights of 17.75 grams for $\mathrm{T}_{1}$ and 12 grams for $\mathrm{T} 2$, revealing that the Physical and chemical factor effects did not affect shrimp growth during the study.

Keywords: Farming; Shrimp; up growth; Salinity.

\section{Introducción}

El cultivo de la especie del camarón blanco (Litopenaus vannamei) a lo largo de los años, implementado en las distintas zonas costeras del occidente de Nicaragua, se expande por la demanda de la producción de alimento, esto manifiesta la importancia de buscar nuevas alternativas de terrenos tierra adentro, para seguir esparciendo este cultivo (Herrera y Martínez, 2009).

Para la consolidación de la camaronicultura como una actividad económicamente viable y ecológicamente sustentable en cultivos tierra adentro, deberán perfeccionarse las técnicas de aclimatación de los organismos acorde al clima de nuestro país, de manera que se logre optimizar y expandir a terrenos no actos para la agricultura por estar sobreexplotados.

Este tipo de cultivo con la utilización de agua subterránea de pozos de baja salinidad ofrece ventajas en comparación con el cultivo tradicional que se realiza en zonas costeras surgiendo como una alternativa para limitar o evitar la propagación de ciertas enfermedades virales que se trasmiten a través de vectores presente en el agua de mar, y que han provocado el colapso en granjas camaroneras de diferentes áreas geográficas (Hernández, 1991). 
Un factor determinante en el crecimiento del camarón en espacios no salitrosos, es su capacidad osmorreguladora, convirtiéndolo en un organismo adaptable a fuentes de agua con bajas salinidades, ya que presenta gran tolerancia a factores ambientales para soportar un intervalo de salinidad entre 0.5- 45 ups. (Gutiérrez, 2001).

De esta manera, el cultivo de camarón blanco en tierra adentro representa una alternativa viable de producción, ya que presenta similitud en los estándares de producción del camarón cultivado con agua de mar, en base a su crecimiento y supervivencia.

\section{Revisión de literatura}

La camaronicultura en el mundo se ha ido incrementando de manera acelerada en las últimas décadas, alcanzando un desarrollo gradual con el cultivo de especies de Litopenaeus vannamei. Desde su inicio hasta el año 1996, cuando la industria del cultivo de camarón marino enfrentó unos de los mayores retos, con la incidencia de enfermedades que llegaron de otras regiones del mundo, las granjas productoras de camarón a gran escala, más que erradicar las enfermedades que azotaban, implementaron un sin número de estrategias que permitieron continuar con el desarrollo y crecimiento de esta actividad (Herrera y Martínez, 2009)

Sin embargo, el crecimiento de esta industria se ve afectado por la falta de tierras aptas para el cultivo de camarón blanco, ya que las zonas marino-costeras disponibles constituyen áreas protegidas en donde importantes comunidades de manglares y biota en general se desarrollan y que, no deberían utilizarse para la expansión de la acuicultura.

Por tales razones el cultivo de camarón blanco en tierra adentro debería ser una nueva alternativa de producción acuícola. Esta alternativa se ha venido implementando desde Asia al menos desde 1987 y en Estados Unidos desde la década de los 90 (Boyd, 2001 citado por Marcillo, 2001).

Estudios realizados en otros países indican que el camarón blanco puede ser cultivado de manera exitosa en tierra adentro con aguas de baja salinidad (o.5 - 5.0 ppm) (Tamayo, 1998). Aunque sin obviar una de las limitantes para desarrollar este tipo de cultivo es la composición iónica de la fuente de agua.

En Guatemala ya existen antecedentes de este tema a través de un estudio realizado en la Escuela de Agricultura de Nororiente (EANOR) en La Fragua, Zacapa en el 2005-2006. Este estudio tuvo por objetivo contribuir al desarrollo de tecnología para la diversificación de la producción agropecuaria en el área rural de Guatemala mediante el cultivo de camarón marino en agua dulce, así como la identificación de los impactos ambientales potenciales de este cultivo. Aun y cuando, los resultados aún no han sido publicados (Marroquín, Valdés, y González, 2012). 
En otros estudios se estimó el crecimiento del camarón blanco a una salinidad de 2\%o en la granja "Los Álamos" ubicada en el margen derecha del río San Lorenzo, Culiacán, Sinaloa, México. Los muestreos fueron a partir de Julio - noviembre del 2002 y con un intervalo semanal.

El cultivo representó un porcentaje de sobrevivencia del $71 \%$, y en peso máximo de $26.8 \mathrm{~g}$ a una tasa de crecimiento de $\mathrm{k}=\mathrm{o}, 8$. Este resultado fue considerado importante debido a que otros autores como Atwood y Cols (2003) señalaron sobrevivencia del $100 \%$ a postlarvas mantenidas a una salinidad de $2 \%$, otros autores fueron; Angulo y Cols (2005) que registraron $73,2 \%$ de sobrevivencia durante 131 días en condiciones de agua de pozo con una salinidad en promedio de $0,8 \%$, Sowers y Cols (2006), quienes a salinidades de 2 y 1\%o, determinaron un 69 y 68\%, mientras, Valenzuela y Cols (2010) con 77\% en agua extraída de pozo, estudios citados por Arzola, Flores, Ceja y Gutiérrez, (2016).

En el estado de Colima en México operan alrededor de 12 granjas con suministro de agua a partir de riego agrícola, operando con sistemas de producción semi - intensivo y alcanzando producciones de 2 toneladas por ha por ciclo con tallas de 14 a 20 gramos (Gutiérrez, 2001).

Mientras que, en Centroamérica, Honduras es el país pionero en el cultivo de camarón tierra adentro obteniendo tasas de crecimiento de 0.51 gramos por semana en salinidades de $2 \mathrm{ppm}$, con sobrevivencia de $55 \%$ y pesos de 5.2 durante 21 días de experimentación. (Meyer, 2004)

Estos por mencionar algunos de los trabajos que a lo largo de los años se han venido comprobando respecto a la adaptabilidad del camarón al cultivo en bajas salinidades y dando respuesta a la población a cerca de opciones de cultivo en sus fincas, cabe destacar que estos mismos trabajos se están realizando continuamente permitiendo mejorar las producciones del cultivo e integrándolo con cultivos agrícolas como ocurre con el cultivo de la tilapia.

\section{Materiales y métodos}

El estudio se realizó en las instalaciones del Laboratorio de Investigaciones Marinas y Acuícola (LIMA, UNAN- LEÓN), ubicado en la comunidad de la Peñitas- Poneloya a $21 \mathrm{Km}$ al suroeste de la ciudad de León, la que se conecta por una carretera pavimentada en perfectas condiciones.

El estudio constó de dos pilas de concretos de $4.4 \mathrm{~m}^{2}$, con un nivel operativo de $1.20 \mathrm{mts}$, el primer tratamiento ( $\mathrm{T} 1$ ): Cultivo de organismos en salinidad óptima, el tratamiento dos ( $\left.\mathrm{T}_{2}\right)$ : Cultivo de organismos en salinidad cercana a o. Cada una con 
capacidad de 5.28 ton de agua; bajo un sistema intensivo de 27 organismos por metro cuadrado. A estas pilas se les proporcionó aireación constante a través de un blower que inyecta el oxígeno por medio de mangueras de $1 / 4$ de pulgadas con piedras difusoras en sus extremos, lo cual ayuda a esparcir el oxígeno por toda la pila.

El agua suministrada durante todo el desarrollo del experimento fue depositada en dos reservorios; un reservorio de $15.75 \mathrm{~m}^{2}$, con capacidad de 54 ton de agua salada. La segunda fuente de agua se encuentra en las instalaciones del Laboratorio de Investigaciones Marinas y Acuícola (LIMA) y es un pozo de agua dulce a una profundidad de 3 metros.

Los camarones utilizados para ambos tratamientos contaron con la salinidad presente en el océano (35 ppm). La aclimatación para el Tratamiento 1 (T1), consistió en adaptar fisiológicamente al organismo al medio que fue sometido.

Se tomaron al azar, con ayuda de una atarraya, un grupo de organismos del recipiente de procedencia, los cuales se contaron, pesaron y adicionado a un pequeño recipiente plásticos que contenía $1000 \mathrm{ml}$ de agua proveniente del laboratorio, a esto se le adicionó $500 \mathrm{ml}$ del agua de estanque esperando 20 minutos para observar su estado fisiológico.

Los organismos resistentes de la aclimatación se adicionaron a su respectiva pila de cultivo a una densidad de siembra de 27 organismos por $\mathrm{m}^{2}$. La aclimatación para el Tratamiento 2 (T2), consistió; en una mezcla de agua de mar y agua de pozo, debido a que el perfil iónico del agua es muy similar al agua del mar sólo que con menor salinidad (Lucena et al., 2006).

Con la ayuda de un chayo se tomaron al azar un grupo de organismo del recipiente de procedencia, los cuales se pesaron y contaron para luego ser adicionados en su respectiva pila de cultivo con una densidad de siembra de 27 organismos por $\mathrm{m}^{2}$.

El procedimiento para la aclimatación de los organismos de agua de mar con 35 ppm a agua de pozo con o ppm de salinidad, se realizó paulatinamente en aproximadamente 6o horas disminuyendo la salinidad a relación de; 2 ppm por cada hora para disminuir la salinidad, desde los 35 ppm a 25 ppm; 2 ppm por cada hora para disminuir 25 ppm de salinidad a 15 ppm; 1 ppm por cada dos horas para disminuir de 15 ppm a 10 ppm; 1 ppm por cada 3 horas para disminuir de 10 ppm a 5 ppm y finalmente 1 ppm por cada cinco horas para disminuir 5 ppm a o ppm de salinidad.

La salinidad fue medida por medio de un refractómetro modelo S10o. Antes de la toma de este parámetro se calibró el refractómetro de la siguiente manera: se coloca una gota de agua de salinidad cero en el prisma del refractómetro, luego se visualiza a contraluz y se observa que la línea azul llegue a cero. Si no es así, con un desarmador 
se ajusta el tornillo en la parte superior hasta ajustar a cero. Este parámetro se monitoreo durante todo el experimento en horas de 6:00 am y 6:00 pm. La Temperatura y el oxígeno fueron medidos por medio de un mismo aparato llamado Oxigenómetro modelo YSI 550A, debido a que este aparato presenta dos sensores que percibe la Temperatura $\left({ }^{\circ} \mathrm{C}\right)$ y el Oxígeno Disuelto $(\mathrm{mg} / \mathrm{L})$ en el agua.

Para la calibración de este equipo se presiona la tecla MODE situada en el centro del aparato, se anota el valor de la salinidad del agua de muestra y la altura sobre el nivel del mar, luego se presiona la tecla de REGRESO e iniciamos la toma de dato, donde se introduce el electrodo del aparato en el agua de cultivo, sumergiéndolo a unos $15 \mathrm{~cm}$ de profundidad, y después de un minuto y medio aproximadamente se muestra el resultado de la lectura. La toma de dato se efectuó dos veces al día (6:00 a.m. y 6:00 p.m.) durante todo el período de experimentación.

El pH se medió por medio de un pH-metro portátil (o de bolsillo) modelo PHep BY HANNA. Para la calibración de dicho aparato se sumerge la sonda de $\mathrm{pH}$ en una solución buffer de $\mathrm{pH} 7$ y debe permanecer en esta solución por algunos minutos para su estabilización. Para la toma de dicho dato se procede a introducir la sonda de $\mathrm{pH}$ en la superficie del agua, esperando hasta que se refleje el resultado. La toma de dato se efectuó dos veces al día (6:00 a.m. y 6:0o p.m.) durante todo el período de experimentación.

Para los muestreos poblacionales se capturaron al azar con ayuda de un chayo, un total de 20 organismos de ambas pilas de experimento. Los organismos capturados se colocaron en un recipiente plástico con agua de las respectivas pilas, estos se pesaron individualmente por medio de una balanza gramera Scout Pro de 500 gramos de capacidad.

Luego todos los pesos obtenidos de los organismos en muestreo de cada pila experimental se suman y se dividen entre el número total de organismo capturado de cada estanque. Todo esto se calcula a través de la siguiente formula.

Para el análisis del Factor de Conversión Alimenticia se tuvo en mano los datos alimenticios de los organismos en cultivo, datos reflejados en las tablas de alimentación de cada pila experimental.

Este cálculo consistió en la suma total del alimento suministrado o mejor dicho alimento acumulado durante las semanas de experimentación entre el peso total ganado semanalmente de los organismos en cultivo o biomasa actual. Para la deducción de este factor se utiliza la siguiente formula. 


\section{Resultados y discusión}

\section{Salinidad}

Los niveles de salinidad durante el período de estudio para ambos tratamientos T1 (Salinidad Óptima) y T2 (Salinidad cercana a cero), presentan una leve tendencia a variar sus fluctuaciones en el tiempo, debido a las variaciones climáticas durante el período de realización de este experimento, obteniendo datos mínimos de 20\% y un máximo de 32\%o en el $\mathrm{T} 1$, en el caso del $\mathrm{T} 2$ el valor mínimo de o\% y un máximo de $2 \%$ durante la primera semana de cultivo.

Con respecto a lo expresado anteriormente y en base al análisis estadístico realizado a través de la prueba de Kolmogorov - Smirnov nos indica que los datos no se distribuyen según una distribución normal por presentar un nivel de significancia menor de 0.05 , la prueba U de Mann - Whitney nos expresa que se da una clara diferencia significativa de la salinidad entre ambos tratamientos, presentando un nivel de significancia menor de 0.05 .

Esta variación o comportamiento de la salinidad se debió en primera instancia a la diferencia marcada de este factor en cada tratamiento, es decir que el estudio al consistir en cultivos de distintas salinidades, la variación de esta en ambos tratamientos iba a ser marcadamente diferente esta salinidad en el tratamiento de salinidad optima ( $\mathrm{T}_{1}$ ) no tuvo repercusiones negativas en el crecimiento ya que los valores de tolerancia para el camarón con respecto a este factor es de 25 y 45\%, según Ponce et al (1997), en cambio Tamayo (1998), nos dice que el crecimiento del camarón en aguas con salinidad cercanas a cero puede ser exitosa siempre y cuando esta cumpla con la cantidad de iones de carbonato de calcio requeridos por el camarón para su óptimo crecimiento. Ver figura $\mathrm{N}^{\circ} 1$. 


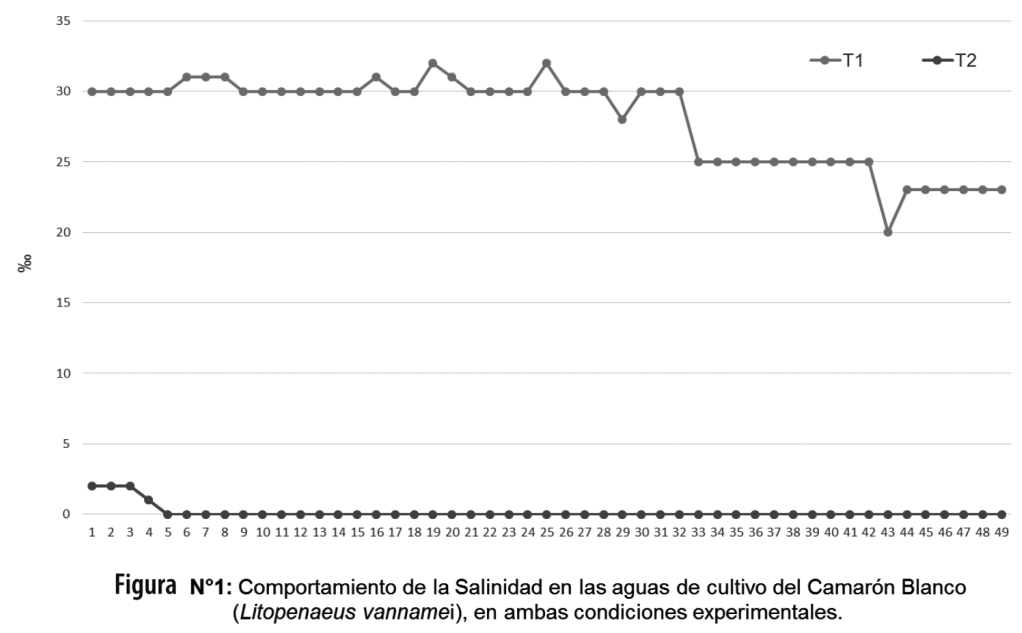

\section{Temperatura}

Los niveles de temperatura durante el período de estudio para ambos tratamientos $\mathrm{T}_{1}$ (Salinidad optima) y T2 (Salinidad cercana a cero), presentan una leve tendencia a variar sus fluctuaciones en el tiempo, debido a variaciones climáticas durante el período de realización de este experimento, obteniendo datos mínimos de $28^{\circ} \mathrm{C}$ y un máximo de $32{ }^{\circ} \mathrm{C}$ en el $\mathrm{T} 1$, en el caso del $\mathrm{T} 2$ el valor mínimo de $27.9{ }^{\circ} \mathrm{C}$ y un máximo de $32.4{ }^{\circ} \mathrm{C}$

Con respecto a lo expresado anteriormente, y en base al análisis estadístico realizado a través de la prueba kolmogorov-Smirnov o Shapiro-Wilks nos indica que no hay normalidad en el comportamiento de los datos por presentar un nivel de significancia menor de 0.05 , la U de Mann-Whitney nos indica que no hay diferencia significativa por presentar niveles mayores de 0.05 .

Los valores de tolerancia con respecto a la temperatura para el camarón fluctúan entre $25-32{ }^{\circ} \mathrm{C}$ según Meyer (2004), por lo que podemos afirmar a través de los valores de temperatura obtenidos durante el ensayo que estas estuvieron dentro de los intervalos óptimos, por lo que no tuvo repercusiones negativas en el crecimiento de los organismos, al contrario se desarrolló como un factor propicio para el crecimiento de los camarones en ambos tratamientos $\mathrm{T}_{1}$ (Salinidad óptima) y T2 (Salinidad cercana a cero), ya que al estar en niveles óptimos la tasa metabólica del animal no se vio influida por este factor. Ver gráfico 2. 


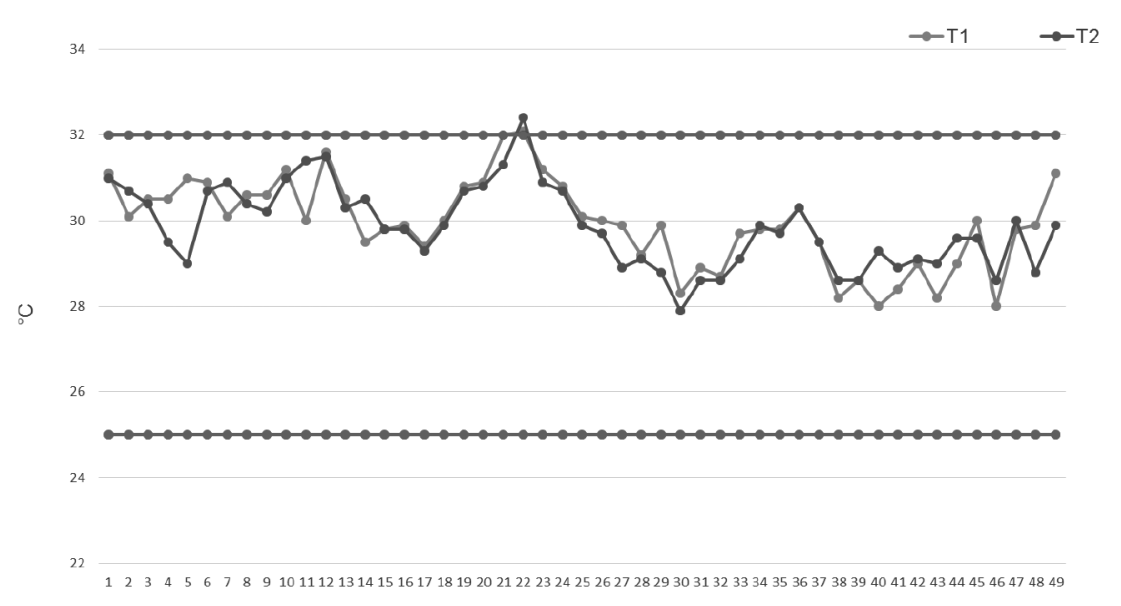

Figura №2: Comportamiento de Temperatura en las aguas de cultivo del Camarón Blanco (Litopenaeus vannamei), en ambas condiciones experimentales.

\section{Oxígeno disuelto}

Los niveles de oxígeno disuelto durante el período de estudio para ambos tratamientos T1 (Salinidad óptima) y T2 (Salinidad cercana a cero), presentan una leve tendencia a variar sus fluctuaciones en el tiempo, debido a las variaciones climáticas presentes durante el ensayo, obteniendo datos mínimos de $1.60 \mathrm{mg} / \mathrm{Lt}$ y un máximo de $5.60 \mathrm{mg} / \mathrm{Lt}$ en el T1, en el caso del T2 el valor mínimo de $2.70 \mathrm{mg} / \mathrm{Lt}$ y un máximo de $6.10 \mathrm{mg} / \mathrm{Lt}$.

Con respecto a lo expresado anteriormente, la prueba kolmogorov-Smirnov o Shapiro-Wilks nos indica que no hay normalidad en el comportamiento de los datos por presentar un nivel de significancia menor de 0.05, la prueba U de Mann Whitney nos expresa que no hay diferencia significativa por presentar niveles mayores de 0.05 .

Según Herrera y Martínez (2009) los valores de tolerancia para el camarón con respecto al oxígeno disuelto son de 3-8 $\mathrm{mg} / \mathrm{Lt}$. De acuerdo con lo anterior, el oxígeno disuelto para ambos tratamientos $\mathrm{T}_{1}$ (Salinidad óptima) y $\mathrm{T}_{2}$ (Salinidad cercana a cero), no tuvo repercusiones negativas en el crecimiento de los organismos, ya que les permitió aprovechar al máximo la energía contenida en el alimento degradado a través del metabolismo, puesto que al estar disponible este gas en el cuerpo de agua los camarones no presentaron alteraciones en la captación de oxígeno a través de la respiración, debido a que el oxígeno es la moneda energética para que se efectué el metabolismo, favoreciendo así su adecuado crecimiento. Ver gráfico No3. 


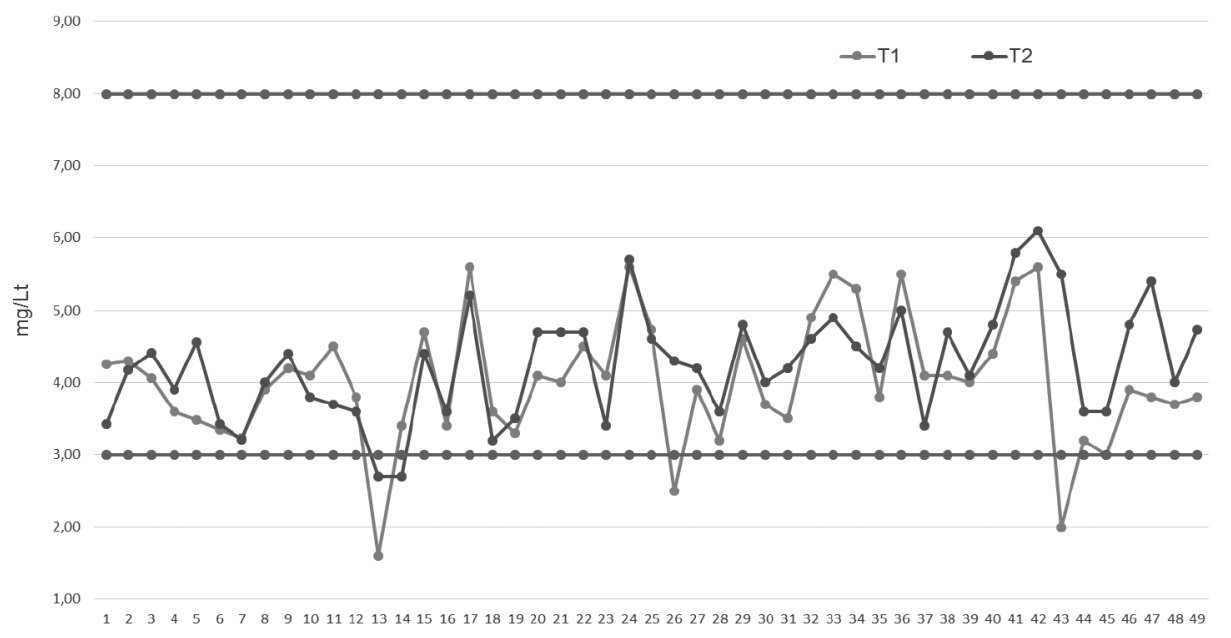

Figura 3: Comportamiento del Oxígeno Disuelto en las aguas de cultivo del Camarón Blanco (Litopenaeus vannamei), en ambas condiciones experimentales.

pH

Los niveles de $\mathrm{pH}$ durante el período de estudio para ambos tratamientos $\mathrm{T} 1$ (Salinidad óptima) y T2 (Salinidad cercana a cero), presentan una leve tendencia a variar sus fluctuaciones en el tiempo, debido al clima que se presentó en el período realización de este experimento, obteniendo datos mínimos de 7.20 y un máximo de 8.70 en el T1, en el caso del T2 el valor mínimo de 7.55 y un máximo de 8.96.

Con respecto a lo expresado anteriormente, la prueba kolmogorov-Smirnov o Shapiro-Wilks nos indica que no hay normalidad en el comportamiento de los datos por presentar un nivel de significancia menor de 0.05, la prueba U de Mann Whitney nos expresa que se da una clara diferencia significativa de la salinidad entre ambos tratamientos presentando un nivel de significancia menor de 0.05 .

Herrera (2012) señala que los valores de $\mathrm{pH}$ fluctúan entre 7,5 - 8,5 para el propicio crecimiento del camarón blanco, de acuerdo con lo anterior y con lo señalado por el autor determinamos que los valores de $\mathrm{pH}$ para el tratamiento $\mathrm{T}_{1}$ (Salinidad óptima) no tuvieron repercusiones negativas en el crecimiento de los organismos. Con respecto al tratamiento T2 (Salinidad cercana a cero), a pesar que los valores de $\mathrm{pH}$ tuvieron fluctuaciones variadas que en este tratamiento no repercutió en gran medida el crecimiento de los camarones, ya que el agua utilizada presentaba carbonato de calcio, ion abundante en agua dulce el cual actúa directamente sobre la alcalinidad de los cuerpos de agua y a su vez influye en el potencial de hidrógeno $(\mathrm{pH})$ en el estanque, 
haciendo más alcalino el medio y por ende el $\mathrm{pH}$ más alto; sin embargo, este elemento iónico no afecto el crecimiento de los camarones debido a que el carbonato de calcio es utilizado por el camarón en aguas cercanas a cero para su crecimiento, ya que este le proporciona la dureza al exoesqueleto en períodos de muda. Ver figura 4.

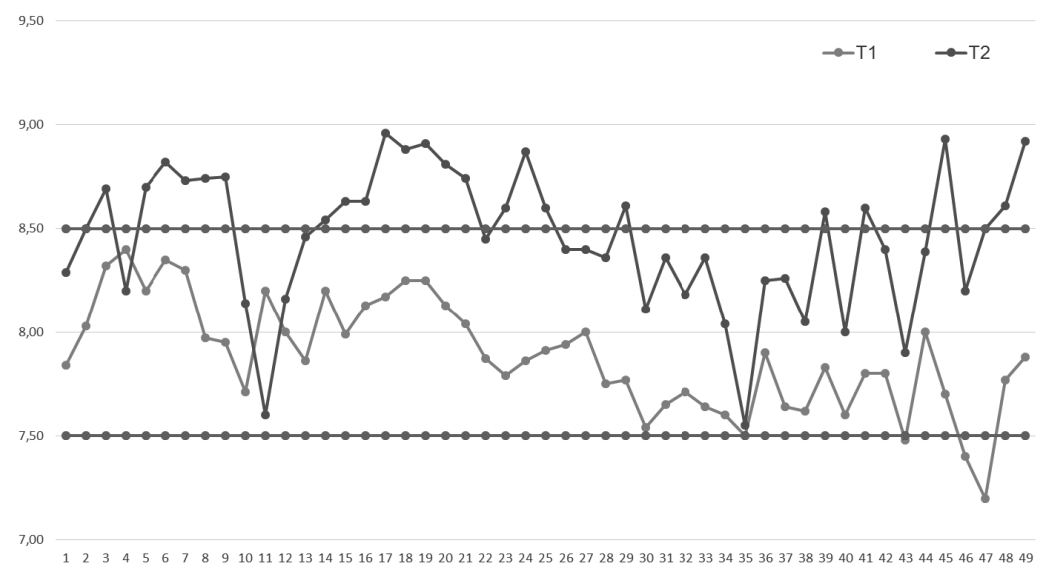

Figura N4: Comportamiento de los niveles de $\mathrm{pH}$ en las aguas de cultivo del Camarón Blanco (Litopenaeus vannamei), en ambas condiciones experimentales.

\section{Crecimiento acumulado}

Con respecto al crecimiento se aprecia en la figura 5 que durante el estudio en ambos tratamientos la ganancia en peso siempre fue ascendente, iniciando con pesos de 3.3 gramos para el T1 (Salinidad óptima) y 1.85 gramos para el T2 (Salinidad cercana a cero), teniendo el $\mathrm{T} 1$ mayor crecimiento que el $\mathrm{T} 2$, obteniendo pesos finales de 17.75 gramos para el $\mathrm{T}_{1}$ y 12 gramos para el T2 con un tiempo de duración de 49 días de cultivo.

Lo expresado anteriormente y en base al análisis estadístico realizado a través de la Prueba Kolmogrov-smirnov se llega a denotar que no hay normalidad en el comportamiento de los datos por presentar un nivel de significancia menor de 0.05 , por lo que se hace la prueba de U de Mann-Whitney presenta un nivel de significancia menor de 0.05 , nos expresa que se da una clara diferencia significativa del crecimiento entre ambos tratamientos.

Martínez (2009) señala que, los valores de crecimiento para sistemas semi-intensivos y con aireación fluctúan o.8 gramos a 2 gramos por semana con pesos finales de 16.5 gramos para un cultivo de 125 días en condiciones óptimas, en cambio para condiciones de bajas salinidad, Marroquín (2012) reporta crecimientos de 0.6 gramos a 0.8 gramos con pesos finales de 14.3 gramos durante 121 días. 
El crecimiento del camarón durante el cultivo en el T1 (Salinidad óptima), todo el tiempo estuvo por encima del crecimiento de T2 (Salinidad cercana a cero) dado que, los factores físico-químicos no tuvieron influencia negativa sobre el metabolismo de los organismos en cultivo y que estos se desarrollaron en un ambiente idóneo, demostrándolo con un peso final por encima del señalado por el autor y en menor tiempo, de igual modo podemos decir que la ganancia en peso del T2 (Salinidad cercana a cero) a pesar que estuvo por debajo del $\mathrm{T}_{1}$ (Salinidad óptima), no tuvo repercusiones negativas con respecto a los factores ambientales y si bien el crecimiento estuvo por debajo del señalado por el autor hay que recalcar que el tiempo es menor que el reportado por Marroquín (2012), dado que si al cultivo se le hubiese dado continuidad efectivamente por el comportamiento ascendente del crecimiento se hubiese superado el peso obtenido por el autor.

Sin embargo, el crecimiento entre tratamientos difiere uno del otro debido a que en el T2 (Salinidad cercana a cero) los organismos tuvieron un mayor gasto energético al momento de la aclimatación, ya que los camarones concentraron sus energías en adaptarse y no generar biomasa durante este proceso marcando significativamente la diferencia entre los dos tratamientos.

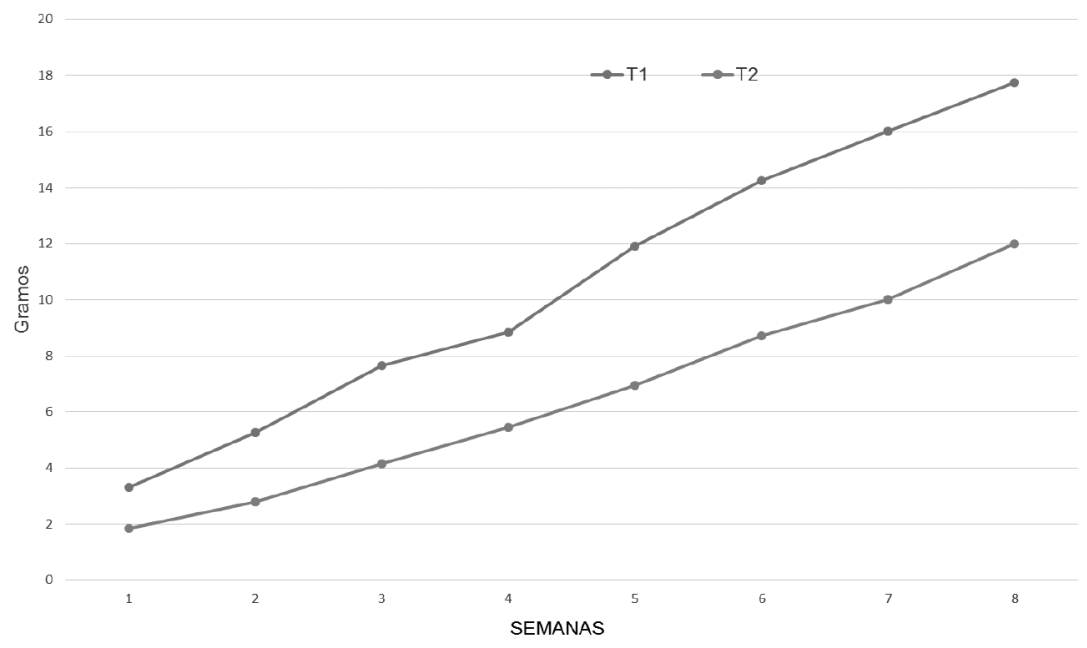

Figura $\mathrm{N}^{\circ}$ : Comportamiento semanal del crecimiento de los camarones blancos cultivados bajo dos condiciones salinidad cercana a cero y salinidad óptima.

\section{Sobrevivencia}

Durante el estudio para el T1 (Salinidad óptima) se mantuvo desde la $1^{\mathrm{a}}$ a la $4^{\mathrm{a}}$ semana con sobrevivencias del 100\%, disminuyendo paulatinamente hasta obtener una sobrevivencia final de $81 \%$; en cambio para el T2 (Salinidad cercana a cero) se presentaron sobrevivencias desde la $2^{\mathrm{a}}$ semana hasta el final del estudio con valores de $99 \%$. 
En base al análisis estadístico con la Prueba Kolmogrov-Smirnov se sabe que los datos no se distribuyen normalmente por presentar un nivel de significancia menor de 0.05 , por lo que se hace la prueba de U de Mann-Whitney que presenta un nivel de significancia menor de 0.05 , lo que indica que existe diferencia significativa del crecimiento entre ambos tratamientos.

Bonilla y Marcial (1994), señalan sobrevivencias de 79.3\% en 120 días de cultivo bajo condiciones óptimas de salinidad, mientras Angulo y Cols (2005), reportan sobrevivencias de $73.2 \%$ en un periodo de 131 días en salinidades de $0.8 \%$. La sobrevivencia en ambos tratamientos superó lo señalado por los autores citados, a pesar del proceso adaptativo a los cuales fueron sometidos los organismos del T2, sin embargo, podemos apreciar que, en el $\mathrm{T}_{1}$, aunque no fue influenciado por los parámetros ambientales en todo el periodo de estudio tuvo un descenso considerable en la población, dando una menor sobrevivencia del $\mathrm{T}_{1}$ con respecto al $\mathrm{T}_{2}$, pero aceptables con respecto a lo que refleja bibliografía.



Figura $\mathrm{N}^{\circ}$ 8: Comportamiento semanal de sobrevivencia de los camarones blancos cultivados bajo dos condiciones salinidad cercana a cero y salinidad óptima.

\section{Factor de Conversión Alimenticia (FCA)}

Los valores de F.C.A para ambos tratamientos: $\mathrm{T}_{1}$ (Salinidad óptima) y T2 (Salinidad cercana a cero) se presentaron por debajo de 1:1, teniendo al final del estudio factores de conversión de 0.5:1 para Salinidad óptima y de 0.6:1 para Salinidad cercana a cero.

A través de la Prueba Kolmogrov-Smirnov podemos saber que, los datos no siguen una distribución normal por presentar un nivel de significancia menor de 0.05 , 
por lo que se hace la prueba de U de Mann-Whitney nos dice que existe diferencia significativa del crecimiento entre ambos tratamientos.

Según Herrera (1999), citado por Martínez (2009), detalla que para camarones con pesos mayores de 10 gramos y en sistemas semi-intensivos el F.C.A no debe ser mayor a 1.5:1. Arzola et al. (2016), reporta los mismos valores que el autor anterior.

Los valores de FCA obtenidos en el estudio son menores en comparación con los valores señalados por los autores en ambas condiciones experimentales, esto gracias a que los factores físicos y químicos, como la calidad de agua en general no influyeron negativamente en el organismo, lo que indica que el alimento suministrado fue transformado en biomasa de manera exitosa.

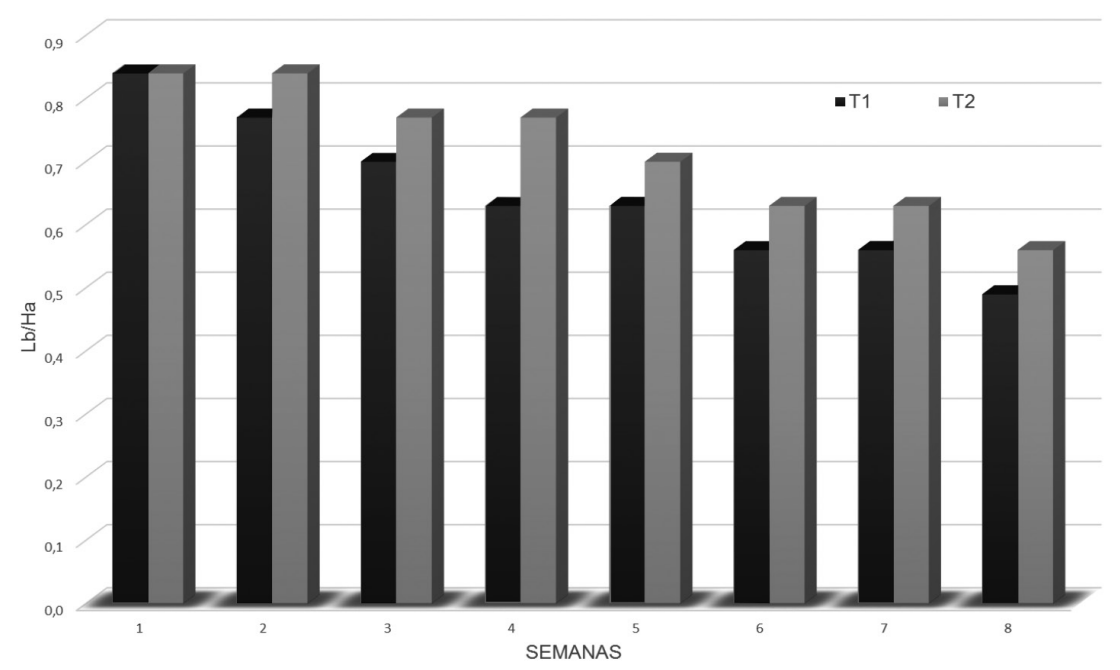

Figura $\mathrm{N}^{\circ} 9$ : Comportamiento semanal del Factor de Conversión Alimenticio de los camarones blancos cultivados bajo dos condiciones salinidad cercana a cero y salinidad óptima.

\section{Conclusiones}

Los factores físicos-químicos obtenidos durante el estudio fueron para el factor salinidad un mínimo de $20 \%$ y un máximo de $32 \%$; temperatura $28{ }^{\circ} \mathrm{C}$ valor mínimo y un valor máximo de $32^{\circ} \mathrm{C}$; oxígeno $1.60 \mathrm{mg} / \mathrm{L}$ valor mínimo a $5.60 \mathrm{mg} / \mathrm{L}$ valor máximo; $\mathrm{pH}$ valor mínimo de 7.20 y máximo de 8.70 , con respecto a la salinidad en el T1 (salinidad óptima) fluctuó entre 32\%o como máximo y 20\%。 como mínimo, por otro lado para el T2 (Salinidad cercana a cero) la salinidad se presentó constante con un valor de o\%; la temperatura $27.9^{\circ} \mathrm{C}$ valor mínimo y un valor máximo de $32.4{ }^{\circ} \mathrm{C}$; oxígeno de $2.70 \mathrm{mg} / \mathrm{L}$ valor mínimo a $6.10 \mathrm{mg} / \mathrm{L}$ de valor máximo; $\mathrm{pH}$ valor mínimo de 7.55 y máximo de 8.96. Los resultados obtenidos en el estudio concuerdan con 
los valores de diferentes autores referenciados razón por la cual denotamos que los factores físico-químicos no repercutieron de forma negativa en el crecimiento de los organismos en cultivo.

Con respecto a los muestreos poblacionales se determinó un peso final de 17.75 gramos para el $\mathrm{T} 1$ y 12 gramos para el T2. La diferencia de peso de los camarones de ambos tratamientos se ve claramente notificada debido a que los organismos del T2 tuvieron un mayor gasto energético al momento de aclimatarse al nuevo medio sometido como es, aguas con salinidades cercanas a o. Sin embargo, los pesos finales fueron aceptables para ser utilizados en la comercialización del sector.

La sobrevivencia final en el estudio fue de un $81 \%$ para el T1; en cambio en el T2 se presentaron sobrevivencias finales del 99\%; los valores de Factor de Conversión Alimenticia para el $\mathrm{T}_{1}$ fueron de 0.5:1 y de 0.6:1 para el T2. Esto significan valores aceptables para la producción y comercialización de este producto.

\section{Agradecimiento}

Esta publicación obtuvo el financiamiento de: El Fondo de Asistencia Internacional de los Estudiantes y Académicos Noruegos (SAIH).

\section{Lista de referencia}

Arzola, J., Flores, L., Ceja, A. y Gutiérrez, Y. (2016). Crecimiento de camarón blanco (Litopenaeus vannamei) en un estanque rústico a baja salinidad. Revista AquaTIC, o(28). Recuperado a partir de: http://www.revistaaquatic.com/ojs/index.php/ aquatic/article/view/194

Boyd, C., 2001 citado por Marcillo, F. (2001). Cultivo de camarón marino tierra adentro con agua de pozo sin adición de sales al agua. Recuperado 8 de junio de 2017, a partir de: https://www.google.com.ni/search?q=cultivo+de+camaron+marino +tierra+adentrro+con+agua+de+pozo+sin+adicion+de+sales+al+agua\&oq=cul tivo+de+camaron+marino+tierra+adentrro+con+agua+de+pozo+sin+adicion+ de+sales+al+agua\&aqs=chrome..69i57.21696joj7\&sourceid=chrome\&ie=UTF-8

Bonilla, E. y Marcial, C. (1994). Estimación de la sobrevivencia de Penaeus vannamei (Boone) en un sistema intensivo en Sinaloa. Oceanología, 1(4), 151-156.

Gutiérrez, M. (2001). Cultivo de camarón en agua dulce: la nueva frontera. Panorama Acuícola, 3(6), 32-33. 
Hernández, A. (1991). Proyecto piloto para el cultivo de camarón costero nutrición/ alimentación. Recuperado a partir de: http://www.fao.org/docrep/field/oo3/ $\mathrm{AC}_{516 \mathrm{~S} / \mathrm{AC} 16 \mathrm{So} \text {.htm }}$

Herrera, C., y Martínez, E. (2009). Guía para el componente curricular Camaronicultura. (Carrera de Ingeniería Acuícola). Universidad Nacional Autónoma de Nicaragua UNAN- LEÓN.

Herrera, C. (2012). Factores físicos y químicos del agua de los estanques camaroneros. (Carrera de Ingeniería Acuícola.). Universidad Nacional Autónoma de Nicaragua UNAN- LEÓN. Facultad de Ciencias y Tecnología.

Marroquín, E., Valdés, M., y González, J. (2012). Potencial del Camarón Marino Litopenaeus vannamei Para el cultivo en agua dulce. (pp. 8-10). Guatemala: Universidad de San Carlos de Guatemala. Dirección General de Investigación Centro de Estudios del Mar y Acuicultura. Recuperado a partir de http://digi.usac.edu.gt/bvirtual/ informes/rapidos2011/INF-2011-29.pdf

Martínez, E. (2009). Producción de camarones marinos a dos densidades de siembra en estanques de concreto utilizando sistema intensivo sin aireación. Las Peñitas, Nicaragua. (Programa Desarrollo Institucional (ASDI/SAREC), Proyecto de Desarrollo de la Investigación para académicos con Ms y PhD. :). Nicaragua.

Meyer, D. (2004). Introducción Acuicultura, Escuela Agrícola Panamericana. Zamorano, Honduras 2004, pg. 31:34, 50:56: http://pdacrsp.oregonstate.edu/pubs/featured_titles/Introduccion\%20Acuacultura.pdf

Ponce, J., Martínez, C., y Ross, L. (1997). The effects of salinity and temperature on the growth and survival rates of juvenile white shrimp, Penaeus vannamei, Boone, 1931. Aquaculture, 157(1-2), 107-115.

Tamayo, A. (1998). Camarón Blanco en agua dulce: una nueva opción. Revista Mazatlán Sinaloa, México, 206-212. 